\title{
A Comprehensive Comparative Evaluation of Pixel by Pixel and Discrete Wavelet Transformation Image Fusion Algorithms
}

\author{
Gurpreet Singh, Gagandeep Jindal \\ Department of Computer Science and Engineering CEC Landran, Mohali, Punjab, India \\ Associate ProfessorDepartment of Computer Science and EngineeringCEC Landran, Mohali, Punjab, India
}

\begin{abstract}
Image Fusion is a process of combining the relevant information from a set of images, into a single image, wherein the resultant fused image will be more informative and complete than any of the input images. There are several image fusion algorithms that are being used by the researchers throughout the globe. Each of the image fusion algorithms has their own advantages and disadvantages which are the main area of concern in this paper. The commonly used image fusion algorithms used are pixel by pixel method, discrete wavelet transformation, Haar transformation, PCA base image fusion method, HIS transform base image fusion, etc. In this paper we will discuss the pixel by pixel method and discrete wavelet transformation image fusion methods. A thorough evaluation of all the above mentioned algorithms is conducted with the help of algorithms and mathematical formulas. These algorithms are compared using three quality metrics namely entropy, standard deviation and quality index. The experimental evaluation is conducted using Matlab 7.The reading produced by image quality metrics, based on image quality of the fused images, were used to access the algorithms.
\end{abstract}

Keywords: - Image Fusion, DWT, Pixel by Pixel method.

\section{INTRODCUTION}

Any piece of information makes sense only when it is able to convey the content across. The clarity of information is important. Image Fusion is a mechanism to improve the quality of information from a set of images. By the process of image fusion the good information from each of the given images is fused together to form a resultant image whose quality is superior to any of the input images. This is achieved by applying a sequence of operators on the images that would make the good information in each of the image prominent. The resultant image is formed by combining such magnified information from the input images into a single image. This is achieved by applying sequence of operators on the images that would make the good information in each of the image prominent. The resultant image is formed by combining such magnified information from the input images into a single image [1].

Medical fusion image is to combine functional image and anatomical image together into one image. This image can provide abundance information to doctor to diagnose clinical disease. A new and improved image can be got if taking into account all the images. Image fusion also has wide application domain in medical diagnosis [2]. Also Image fusion provides an effective way of reducing this increasing volume of information while at the same time extracting all the useful information from the source images. Multi-sensor data often presents complementary information about the region surveyed, so image fusion provides an effective method to enable comparison and analysis of such data. However, image fusion does not necessarily imply multi-sensor sources, there are interesting applications for both single-sensor and multisensory image fusion [9]. The rest of the paper is organized as follows. Image Fusion algorithms are explained in section II. Image quality metrics are discussed in section III. Experimental results and their discussions are presented in section IV. Conclusion and future proposal is given in section V.

\section{IMAGE FUSION ALGORITHMS}

\section{A. Pixel by Pixel Image fusion method}

The pixel-level image fusion work assumes that the input images meet a number of requirements. Firstly, input images must be of the same scene, i.e. the fields of view of the sensors must contain a spatial overlap. Furthermore, inputs are assumed to be spatially registered and of equal size and spatial resolution. In practice, size and resolution constraints are often satisfied by resampling one of the input images. Another important consideration in pixel-level fusion is the number of input images and the color characteristics of the input and output images. This paper addresses the topic of pixel-level fusion with two monochrome input images and a monochrome fused output image. However, all the work can be easily extended to accommodate a higher number of inputs and explanations of this are provided where appropriate. Steps of our proposed method of the pixel based image fusion are explained below: 
Step 1: Read the set of multi-focus images i.e. here in our proposed algorithm we have consider two images which are of same size (registered images).

Step 2: Alpha Factor can be varied to vary the proportion of mixing of each image.

- With Alpha Factor $=0.5$, the two images are mixed equally.

- With Alpha Factor $<0.5$, the contribution of background image will be more.

- With Alpha Factor $>0.5$, the contribution of foreground image will be more.

Step 3: Perform element by element multiplication of image array with alpha factor for foreground image and multiply Complement of alpha factor with image array of background image.

Step 4: Now perform pixel by pixel intensity value comparison and find the maximum intensity value.

Step 5: This value is consider for the final output image.

Step 6: Finally display the fused image contains all the high intensity value of the pixel.

\section{B. Discrete Wavelet Transformation Method}

Wavelet transform has been greatly used in many areas, such as texture analysis, data compression, feature detection, and image fusion. In this section, we briefly review and analyze the wavelet-based image fusion technique. The DWT can be interpreted as signal decomposition in a set of independent, spatially oriented frequency channels. The signal $\mathrm{S}$ is passed through two complementary filters and emerges as two signals, approximation and Details. This is called decomposition or analysis. The components can be assembled back into the original signal without loss of information. This process is called reconstruction. The mathematical manipulation, which implies analysis and synthesis, is called discrete wavelet transform and inverse discrete wavelet transform. An image can be decomposed into a sequence of different spatial resolution images using DWT. Wavelet based techniques for fusion of 2-D images is described here. In all wavelet based image fusion techniques the wavelet transforms $\mathrm{W}$ of the two registered input images I1 $(\mathrm{x}, \mathrm{y})$ and some kind of fusion rule $\varnothing$ as show in below equation.

$$
\mathrm{I}(\mathrm{x}, \mathrm{y})=\mathrm{W}-1(\varnothing(\mathrm{W}(\mathrm{I} 1(\mathrm{x}, \mathrm{y})), \mathrm{W}(\mathrm{I} 2(\mathrm{x}, \mathrm{y})))
$$

In the case of wavelet transform based fusion method, all respective wavelet coefficients from the input images are combined using the fusion rule $\varnothing$. Since wavelet coefficients having large absolute values contain the information about the salient features of the images such as edges and lines, a good fusion rule is to take the maximum of the corresponding wavelet coefficients.

Steps of wavelet based image fusion are explained below:

Step 1: Read the set of multi-focus images i.e. here in our proposed algorithm we have consider two images which are of same size (registered images).

Step 2: Apply wavelet decomposition on both the images with the use of Daubechies filter.

Step 3: Extracts from the wavelet decomposition structure [C, S] the horizontal, vertical, or diagonal detail.

Step 4: Perform average of approximation coefficients of both decomposed images.

Step 5: Compare horizontal, vertical and diagonal coefficient of both the images and apply maximum selection scheme to select the maximum coefficient value by comparing the coefficient of the two images. Perform this for all the pixel values of image i.e. $\mathrm{m} \times \mathrm{n}$.

Step 6: Now apply wavelet decomposition on both the images with the use of Daubechies filter.

Step 7: Display the final fused image.

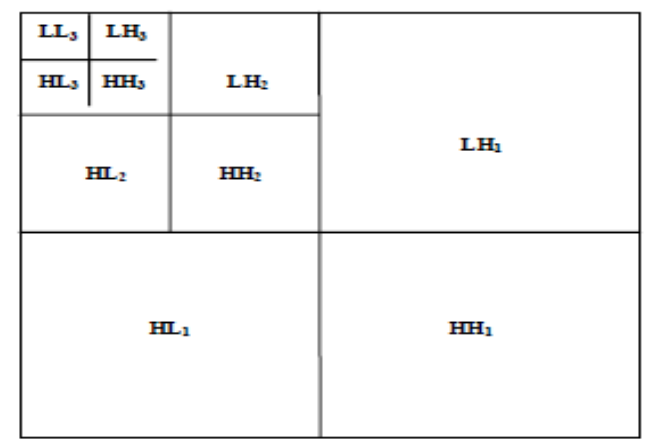

Figure 1: Decomposition of Image using DWT 


\section{III.}

\section{IMAGE QUALITY METRICS}

The Quality metrics are a measure of performance of the image fusion methods used. Image quality is a characteristic of an image that measures the perceived image degradation as compared to an ideal image. The following quality metrics are used in this paper to describe the quality of the image:

1. Standard deviation (represented by the symbol sigma, $\sigma$ ) shows how much variation or dispersion exists from the average (mean), or expected value. A low standard deviation indicates that the data points tend to be very close to the mean; high standard deviation indicates that the data points are spread out over a large range of values.

2. Entropy is a statistical measure of randomness that can be used to characterize the texture of the input image.

3. Image Quality index, which is easy to calculate and applicable to various image processing applications. Instead of using traditional error summation methods, the proposed index is designed by modeling any image distortion as a combination of three factors: loss of correlation, luminance distortion, and contrast distortion.

\section{EXPERIMENTAL SETUP AND RESULT}

In this section we present the experiments and their results along with their discussions. We fuse the selected sample of input images and the quality of the same was accessed using the quality metrics given in the above section. The test set for this evaluation experiment image randomly selected from the internet. Matlab 7.0 software platform is use to perform the experiment. The PC for experiment is equipped with an Intel P4 2.4GHz Personal laptop and 2GB memory. The scheme is tested using ordinarily image processing. From the simulation of the experiment results, we can draw to the conclusion that this method is robust to many kinds of Fused images.

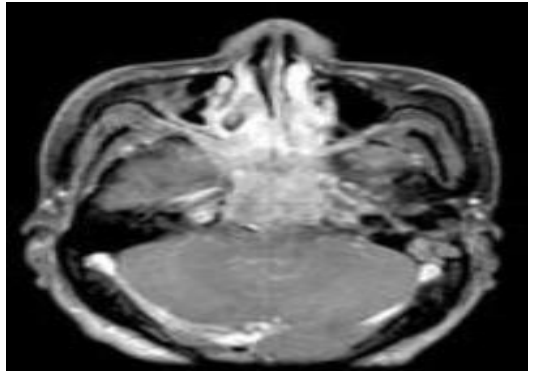

(a)

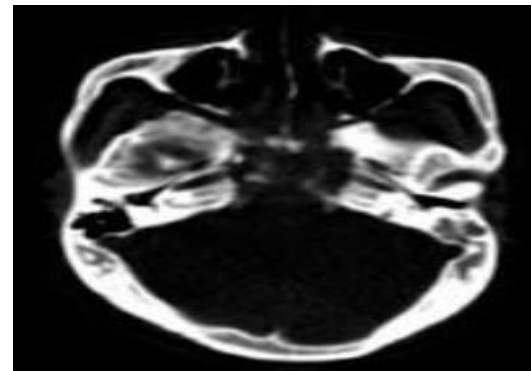

(b)

Figure 2: (a) \& (b) Two sample medical Images from same scene selected for Image fusion

The above two sample images were fused using Matlab 7.0 and the results were used to analyze the quality on the basis of the quality metrics. The resultant images are shown ahead by using both the methods i.e. Pixel by Pixel method and DWT method. First the result of fusion using Pixel by Pixel fusion method is shown in Figure 3 along with the value of results based on the quality metrics.

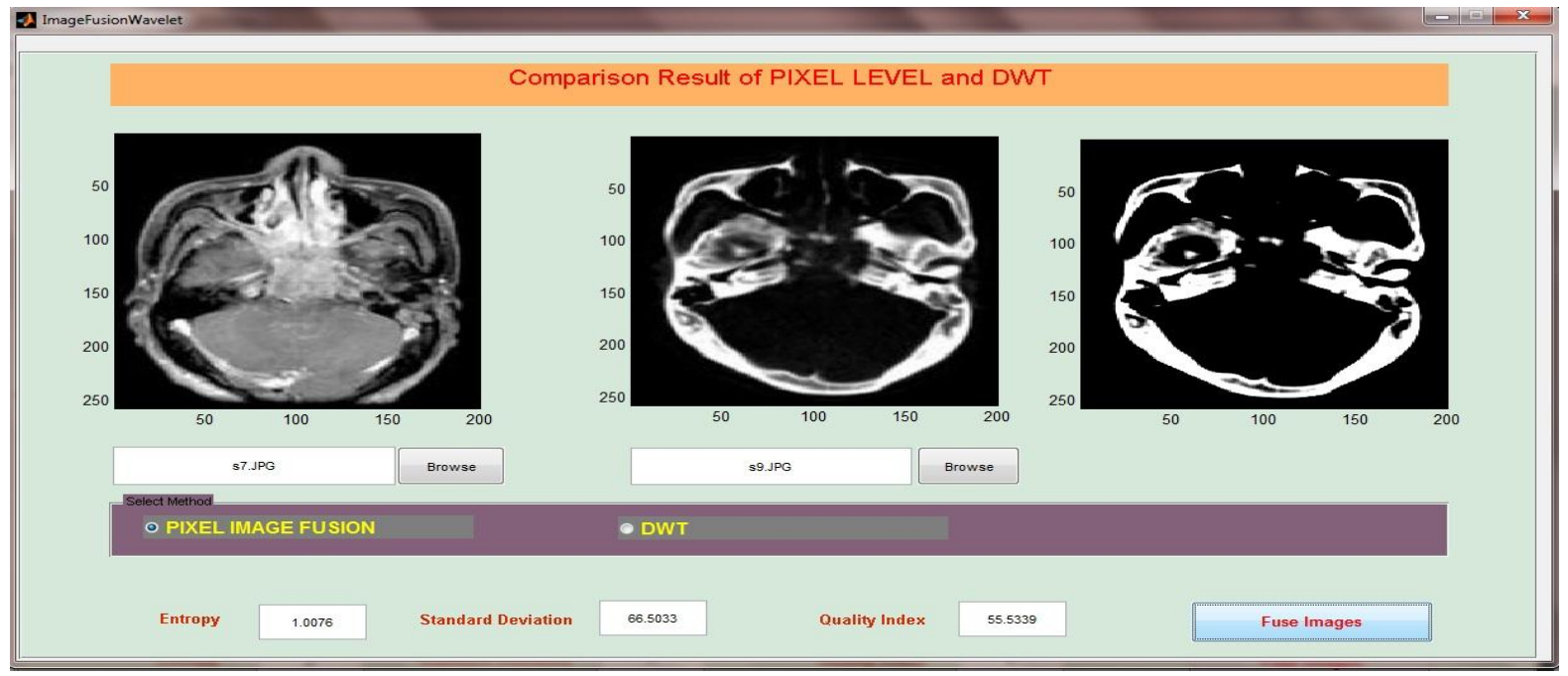

Figure 3: Experiment Result of Pixel by Pixel Image Fusion Method 
The second method used for fusing the two images is DWT method. The resultant image and the value of quality metrics is shown below in Figure 4. The

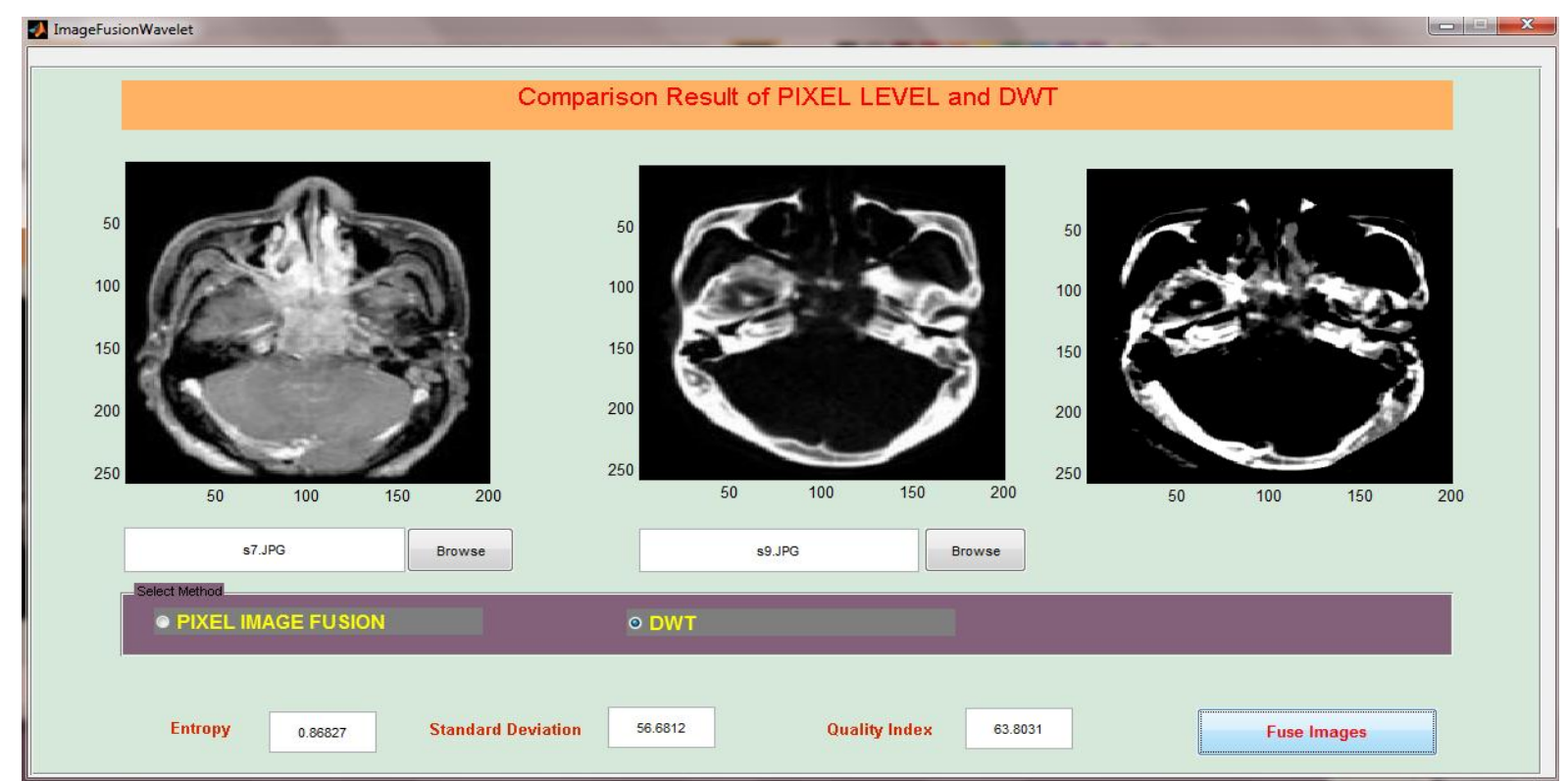

Figure 4: Experiment Result of Fusion by Using Discrete Wavelet Transformation Method

The value of entropy for Pixel by pixel method is 1.0076 and it is more than the value of entropy for DWT method which is 0.86827 . The Figure 5 shows the graphical representation of entropy for both the methods.

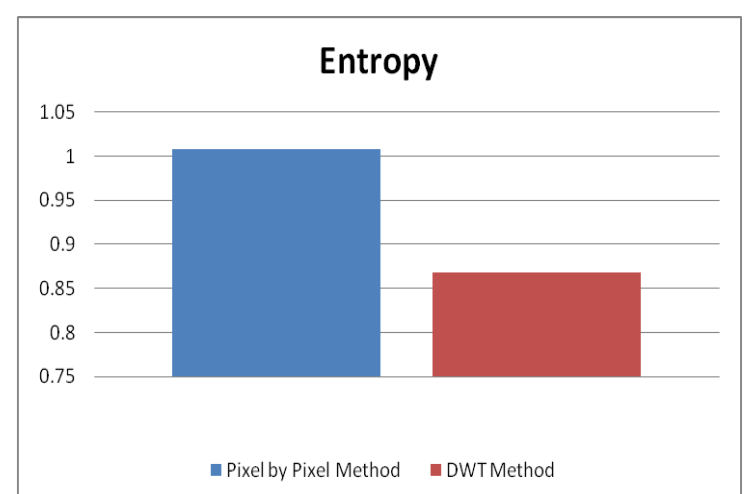

Figure 5: Entropy for Pixel by pixel and DWT methods

The value of standard deviation for Pixel by pixel method is 66.5033 and it is more than the value of standard deviation for DWT method which is 56.6812. The Figure 6 shows the graphical representation of standard deviation for both the methods.

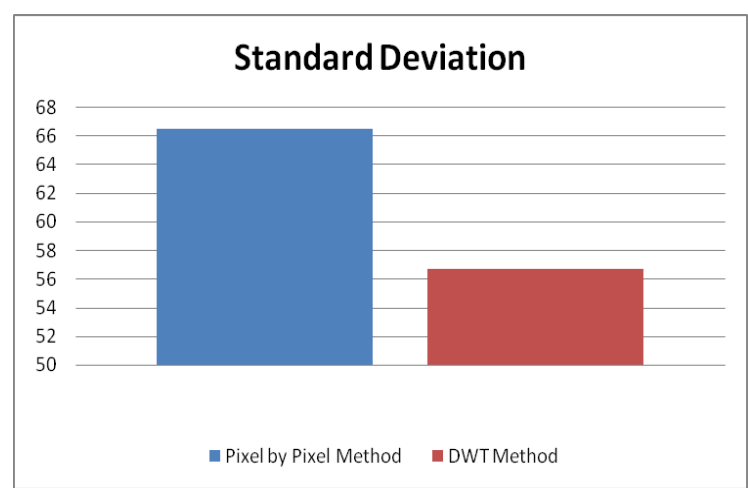

Figure 6: Entropy for Pixel by pixel and DWT methods 
The value of Quality Index for Pixel by pixel method is 55.5339 and it is less than the value of quality index for DWT method which is 68.8031 . The Figure 7 shows the graphical representation of quality index for both the methods.

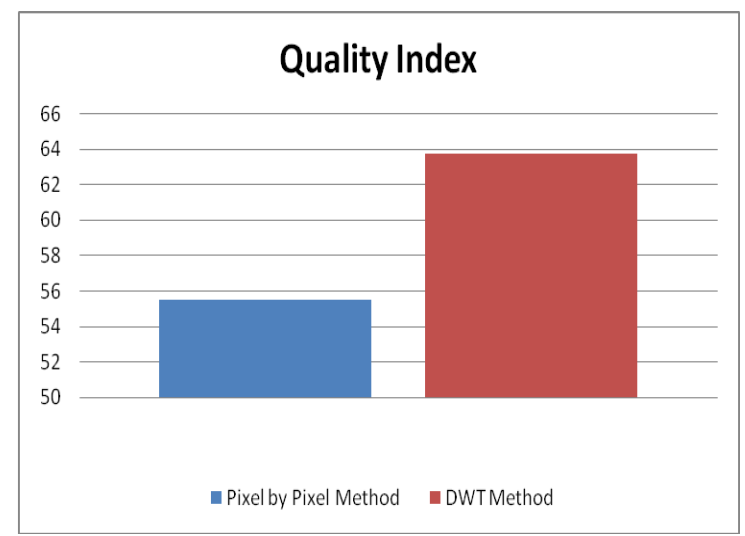

Figure 7: Entropy for Pixel by pixel and DWT methods

The below given Table 1 shows the comparison of pixel by pixel Image fusion method and Discrete Wavelet Transform method. The below given table enable us to analyse the image fusion results with better perception.

Table -1 Result analysis of Pixel by Pixel and DWT method

\begin{tabular}{|c|c|c|}
\hline & Pixel by Pixel Method & DWT \\
\hline Entropy & $\mathbf{1 . 0 0 7 6}$ & $\mathbf{0 . 8 6 8 2 7}$ \\
\hline Standard deviation & $\mathbf{6 6 . 5 0 3 3}$ & $\mathbf{5 6 . 6 8 1 2}$ \\
\hline Quality Index & $\mathbf{5 5 . 5 3 3 9}$ & $\mathbf{6 3 . 8 0 3 1}$ \\
\hline
\end{tabular}

\section{CONCLUSION}

In this paper we have experimentally analyzed two image fusion techniques namely pixel by pixel image fusion method and DWT method using Entropy, Standard Deviation and Quality Index Image Metrics. These experiments were conducted using Matlab 7.0. Depending upon the purpose of a given application, (1) some users may desire a fusion result that shows more detail in color, for better image interpretation or mapping; (2) some may desire a fusion result that improves the accuracy of digital classification; and (3) some others may desire a visually beautiful fused color image, solely for visualization purposes. Therefore, distinct techniques for mapping oriented fusion, classification-oriented fusion, and visualization-oriented image are in demand.

Following conclusions have been drawn from experimental results:

- DWT (Discrete wavelet Technique) have better Image quality Index as compare the Pixel by Pixel Image Fusion Method

- DWT (Discrete wavelet Technique) have lower Standard deviation as compared the Pixel by Pixel Image Fusion Method

- DWT (Discrete wavelet Technique) have lower Entropy as compared the Pixel by Pixel Image Fusion Method

The overall evaluation shows that DWT method is far better than Pixel by Pixel image fusion method.

In future study we propose a new modified image fusion method i.e. Modified Haar Wavelet Transformation method which will give better image fusion than the previous methods.

\section{REFERENCE}

[1] Shivsubramani Krishnamoorthy. and K P Soman (2010), "Implementation and Comparative Study of Image Fusion Algorithms”, International Journal of Computer Science \& Communication, Volume 9No.2, November 2010.

[2] S.Mary Praveena and Dr.ILA.Vennila (2009) "Image Fusion By Global Energy Merging", International Journal of Recent Trends in Engineering, Vol 2, No. 7, November 2009 
[3] V.P.S. Naidu and J.R. Raol (2008) "Pixel-level Image Fusion using Wavelets and Principal Component Analysis", Defence Science Journal, Vol. 58, No. 3, May 2008, pp. 338-352 Ó 2008, DESIDOC

[4] Chetan K. Solanki and Narendra M. Patel "Pixel based and Wavelet based Image fusion Methods with their Comparative Study", National Conference on Recent Trends in Engineering \& Technology

[5] M. Jaya Manmadha Rao and Dr.K.V.V.S.Reddy(2011) "Image Fusion Algorithm for Impulse Noise Reduction in Digital Images", Global Journal of Computer Science and Technology Volume 11 Issue 12 Version 1.0 July 2011

[6] Prakash NK "IMAGE FUSION ALGORITHM BASED ON BIORTHOGONAL WAVELET", International Journal of Enterprise Computing and Business Systems ISSN (Online) : 2230-8849 http://www.ijecbs.com Vol. 1 Issue 2 July 2011

[7] C. POHL and J. L. VAN GENDEREN "Multisensor image fusion in remote sensing: concepts, methods and applications", int. j. remote sensing, 1998, vol. 19, no. 5, 823-854

[8] Melkamu H. Asmare1, Vijanth S. Asirvadam2, Lila Iznita3 and Ahmad Fadzil M. Hani4 "Image Enhancement by Fusion in Contourlet Transform", international Journal on Electrical Engineering and Informatics - Volume 2, Number 1, 2010

[9] Anjali Malviya1, S. G. Bhirud2 "Image Fusion of Digital Images”, International Journal of Recent Trends in Engineering, Vol 2, No. 3, November 2009

[10] Umesh C. Pati, Pranab K. Dutta and Alok Barua "Feature Detection of an Object by Image Fusion", (C2010 International Journal of Computer Applications (0975 - 8887) Volume 1 - No. 1 